\title{
Stress fractures presenting as tumours: a retrospective analysis of 22 cases: reply to Agarwal and Gulati
}

\author{
A. Fottner • C. Birkenmaier
}

Published online: 6 August 2008

(C) Springer-Verlag 2008

We greatly appreciate the letter of Dr. Anil Agarwal and Dr. Divesh Gulati and their insightful comments expanding on our findings.

Regarding the clinical findings of stress fractures, it is correct that atypical presentations are frequent. For our study we wanted to choose the most common symptom as the inclusion criterion. Even if other findings like swelling and signs of inflammation may be seen, the most frequent symptom at onset is clearly pain [4]. In the mentioned case reports, pain was also the leading symptom $[1,3]$.

Regarding our findings, it must be taken into account that we evaluated unusual cases of stress fractures in which the diagnosis could not be made after performing X-rays and magnetic resonance imaging (MRI). On the MRI scans, unspecific signal enhancements without fracture lines, also suspicious for tumour-like lesions, were present. According to the classification of Fredericson et al. [2], these findings would correspond to grade 2 and grade 3 stress fractures. Since in these cases the differentiation from neoplastic lesions also could not be made on clinical grounds, further examinations were needed. Computed tomography (CT) scans turned out to be most helpful in these cases to identify the stress fracture.

The orientation of the fracture lines perpendicular to the cortex and the major weight-bearing trabeculae is a helpful sign in many cases, but not always reliable as is demonstrated in Fig. 3b of our paper.

\section{References}

1. Daffner RH, Martinez S, Gehweiler JA, Harrelson JM (1982) Stress fractures of the proximal tibia in runners. Radiology 142:63-65

2. Fredericson M, Bergman AG, Hoffman KL, Dillingham MS (1995) Tibial stress reaction in runners. Correlation of clinical symptoms and scintigraphy with a new magnetic resonance imaging grading system. Am J Sports Med 23(4):472-481

3. Lam HS, Wong WC (1993) Stress fracture of tibia that simulates malignancy: a case report. J Hong Kong Med Assoc 45(4):302-304

4. Lassus J, Tulikoura I, Konttinen YT, Salo J, Santavirta S (2002) Bone stress injuries of the lower extremity: a review. Acta Orthop Scand 73(3):359-368 s00264-008-635-7.

A. Fottner $(\bowtie) \cdot$ C. Birkenmaier Orthopedics, Klinikum Großhadern,

Marchioninistr. 15,

81377 Munich, Germany

e-mail: andreas.fottner@med.uni-muenchen.de 